\title{
Design and Research of Thermal Transfer Label Printer Testing System Based on Virtual Instrument Technology
}

\author{
Yang $\mathrm{Xu}^{1, \mathrm{a}}$, Xue Dong ${ }^{2, \mathrm{~b}}$ and Jingmei Cheng ${ }^{3, \mathrm{c}}$ \\ ${ }^{1}$ School of Automation, Chongqing University of Posts and Telecommunications, ChongQing \\ 400065, China; \\ ${ }^{2}$ School of Automation, Chongqing University of Posts and Telecommunications, ChongQing \\ 400065, China. \\ ${ }^{3}$ School of Automation, Chongqing University of Posts and Telecommunications, ChongQing \\ 400065, China. \\ axuyang@cqupt.edu.cn, ${ }^{b} 576259956 @ q q . c o m,{ }^{c}$ 1007004045@qq.com
}

Keywords: Testing System, Thermal Transfer Label Printer, Virtual Instrument Technology, LabVIEW

\begin{abstract}
This paper describes a testing system of thermal transfer label printer based on virtual instrument technology. The hardware of this system uses data acquisition card, signal condition board, while its software is developed on LabVIEW. This system can realize the automated testing for various features of thermal transfer label printer. With simple structure, convenient operation, high stability and friendly interface, it breaks through the limitation due to the traditional testing methods, greatly improving the detection efficiency and precision. Meanwhile, it reduces the influence of human factors and provides an effective automation testing solution for relative printer analysis.
\end{abstract}

\section{Introduction}

With the rapid development of various industries, label has become one of the necessary identifications of goods. So the corresponding industries put forward more and more requirements on label printing devices, which are becoming increasingly diversified. How to ensure the efficiency and stability of the label printer is one of the important issues people care about. Thermal transfer label printer is widely used in various fields with unique ways of working. Testing system for thermal transfer label printer is an important part and an essential means to ensure stability and efficiency, while the traditional testing system has low precision on measurement with complicated operation. Thus, many experts and scholars have done a large number of theoretical research and practical application on testing system for a long time.

The traditional testing method is manual testing, which uses many testing equipments, making complex operations, long testing period, and the testing accuracy is low, thus it cannot fulfill requirements. In a word, it is necessary to propose a set of high-speed automated effective testing solution to the above problems.

\section{Design of testing system for thermal transfer label printer}

Requirements of testing system for thermal transfer label printer. Through summarizing all kinds of automation testing systems at home and abroad, this paper has achieved design of software platform by LabVIEW, completed the measurement and control requirements for thermal transfer label printer. This testing system can get relevant working parameters, analyze the working process and locate the fault position. Hardware system acquires and detects the status signal of the main modules of thermal transfer label printer. Software system implements the analysis of the signals, the performance and the functions. The whole testing system mainly completes the following functions: 
1)Acquiring key point data signals of thermal transfer printer module, monitoring the running state signals for every point.

2)Transferring signals through data acquisition device to PC, using LabVIEW for data processing and analysis, fault diagnosis and position.

3)Judging work status, generating testing diagnosis report, outputting related testing diagnosis results.

\section{The hardware structure}

Hardware structure consists of multifunctional data acquisition card, the corresponding signal conditioning circuit. They respectively connect to the PC and the thermal transfer label printer, which constitute a functional testing system, as shown in figure 1.

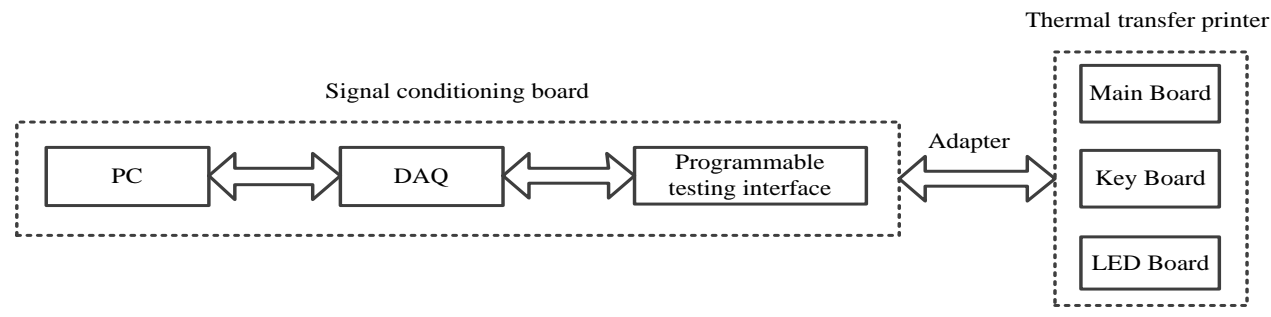

Fig. 1 Hardware structure of testing system

\section{The testing system software design}

The software architecture. This testing system is modular programming, each module independently implements parameter configuration, hardware configuration, data processing, error analysis, generate reports, and other functions, characterized by multiple processes run in parallel. At the same time, LabVIEW graphical programming language can be efficiently used to write a friendly operation interface meeting the need and requirements. The software architecture of testing system is shown in figure 2.

\begin{tabular}{|c|c|c|c|c|}
\hline Data Layer & & \multicolumn{2}{|c|}{ Access Database } & \\
\hline $\begin{array}{l}\text { Application } \\
\text { Layer }\end{array}$ & $\begin{array}{c}\text { Parameter } \\
\text { Configuration }\end{array}$ & $\begin{array}{l}\text { Equipment } \\
\text { Self-check }\end{array}$ & $\begin{array}{c}\text { Report } \\
\text { Management }\end{array}$ & $\begin{array}{c}\text { Function } \\
\text { Verification }\end{array}$ \\
\hline $\begin{array}{l}\text { Presentation } \\
\text { Layer }\end{array}$ & & \multicolumn{2}{|c|}{ Interactive Interface } & \\
\hline System Layer & & \multicolumn{2}{|c|}{ Data Acquisition Card Driver } & \\
\hline
\end{tabular}

Fig. 2: The software architecture

Configuration information module read the configuration information from .ini or .cfg configuration files; distribute the port for related testing projects, in this way can avoid wrong operation on ports. When creating new testing requirements, it can be directly by modifying in the configuration file, to adjust the distribution of the test port, it don't have to be done in program, greatly improving the stability of the testing system.

Functional test module, a signal processing and analysis module, acquire each kind of digital or analog signal, make time-domain analysis or frequency spectrum analysis on the measured point, dynamically display the real-time data, test results and refresh.

Fault diagnosis module, show the correct information stored in the database and test data information with the same variable parameters in the same table, with different colors and lines to distinguish, to compare for users, and to complete the fault isolation and location.

Test diagnostic report, combined with Access, SQL database, store test related information includes an excitation signal, location and scope of the test, the correct results and prompt information. It can write, read, view and import the report by LabVIEW, showing the relevant testing documentations in Word, facilitating to summary about the overall design and production, analyzing the error part with greater probability, making an appropriate adjustments. 
The software design. System combine producer/consumer design pattern with events structure, making the key test, the LED test, the main board test run independently, not interface each other. At the same time, the queue cache mechanism makes the whole testing system more rational; the state machine design pattern is adopted to specify the main board test execution order due to its various and correlation points and signals. This can be clear at a glance and easy to find errors, so that it can improve the system scalability and versatility. Main program flow chart of testing system is shown in Figure 3.

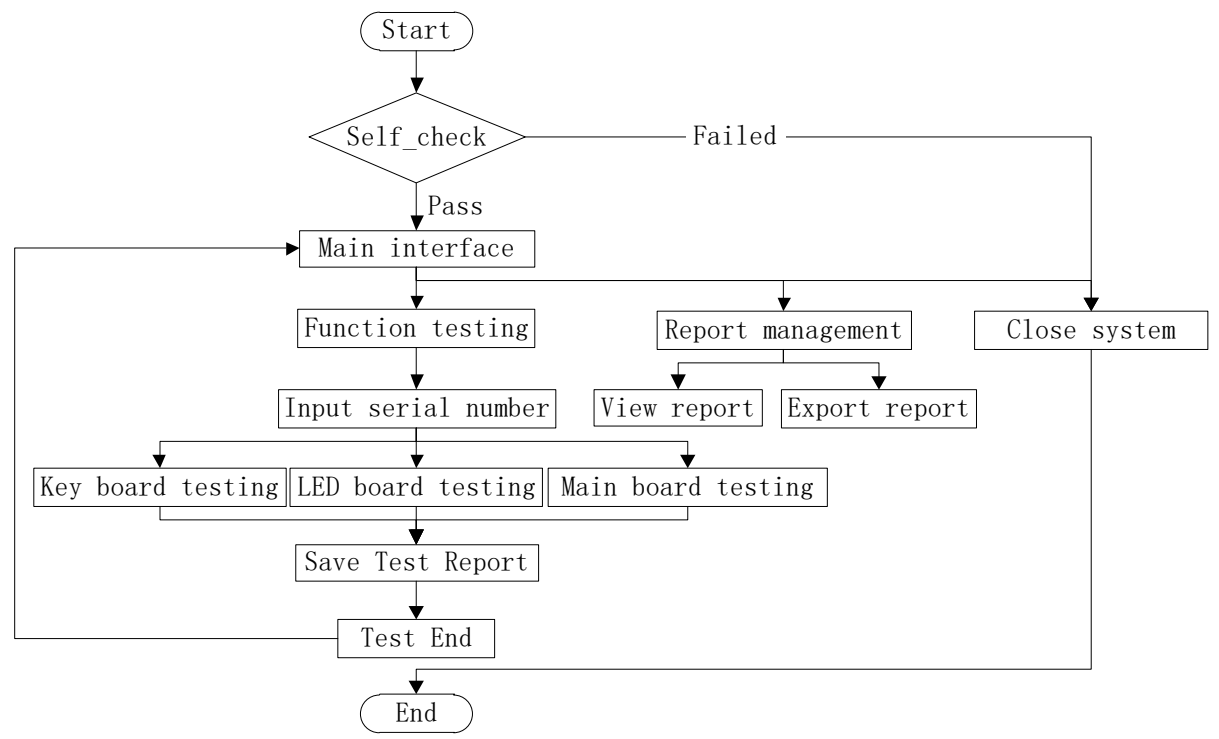

Fig. 3: Main program flow chart of testing system

Enter the system, firstly do self-checking, which is used to detect whether the data acquisition card and signal conditioning board connection is normal. If it goes wrong, then returns to the start interface program, closing the system, reconfirming the hardware connection and so on; else it goes correct, the system then goes into the function testing interface.

1)Key board testing: acquiring and monitoring the corresponding voltage level. When the button is pressed, program will judge whether its voltage level has changed. The testers get the testing results by observing the lights flashing on interface.

2)LED board testing: software programs control to generate specific digital signal as input for LED input pin, then judge the testing result by observing the flashing of LED.

3)Main board testing: software programs control relay power-on, controlling the printer turn-on, simulating the button, and then capturing signal status of main board key points, and analyzing, displaying and saving the test results. Here taking the CANCEL_BUTTON testing as an example, program controls relay power-on, simulating power pressing motion in 3s, then detecting SPI_DATA and SPI_CLK whether there is a pulse output, and determining the CANCLE_BUTTON function is correct, in the same time, showing the testing result and store it in database. The block diagram is shown in figure 4.

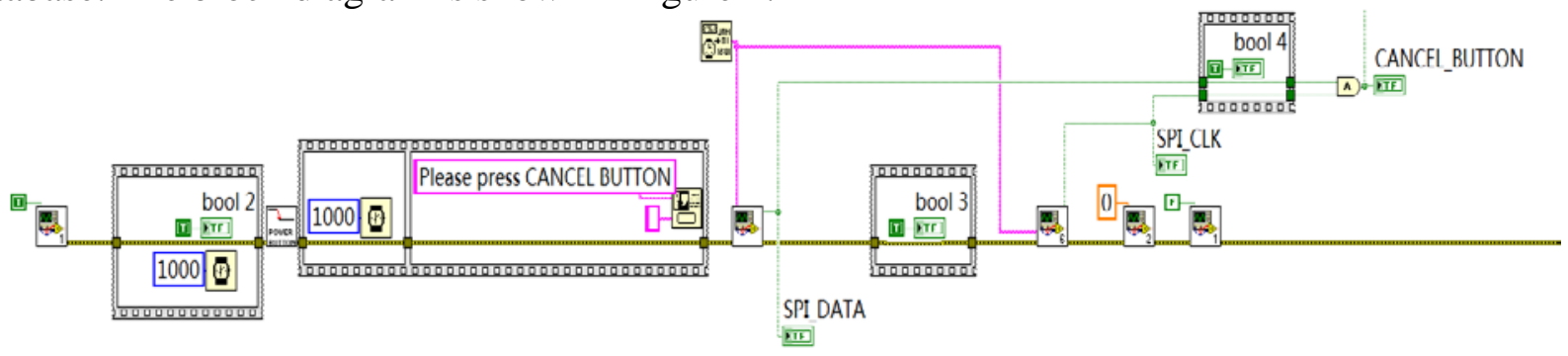

Fig. 4: the block diagram of cancel button testing

\section{Verification of the testing system}

This system is used in the actual production streamlines for testing on 1000 thermal transfer label printers (P1200), the average time and efficiency for testing is shown in table 1. 
Table 1: The comparison chart of test time and test efficiency

\begin{tabular}{ccc}
\hline Testing method & $\begin{array}{c}\text { Average testing time } \\
(\mathrm{min})\end{array}$ & $\begin{array}{c}\text { Testing efficiency } \\
(\%)\end{array}$ \\
\hline Traditional manual test & 47 & 96 \\
Testing system based on virtual instrument technology & 5.75 & 97.8 \\
\hline
\end{tabular}

\section{Summary}

In this paper, by analyzing the working principle and characteristics of thermal transfer label printer, according to the test requirements, finishes testing on 35 specific items, and completes the design of thermal transfer label printer based on virtual instrument testing system. This testing system is equipped with position machine, intuitively displaying the test results, and effectively locating the fault, and recording. There will be no residual problems, making up for the deficiency of the traditional testing methods. Experiments and practices show it is reasonable for this testing system, with high stability, reliability and engineering practice significance, not only shorten the testing cycle and testing cost, but meet the practical production requirements.

\section{Acknowledgments}

It is a project supported by Graduate Innovative Education Key Program in Chongqing University of Posts and Telecommunication, and the project number is Y201308.

\section{References}

[1] T. VO, Z. Wang, et al, Design for board and system level structural test and diagnosis, in Proc. ITC, (2006) 409-418.

[2] Z. Wang, X. Gu, et al. System and method for executing functional scanning in an integrated circuit environment, field with the U.S. Patent Office, 2010.

[3] Fangming Ye, et al. Adaptive Board-Level Functional Fault Diagnosis Using Decision Trees, Test Symposium (ATS), IEEE 21st Asian, (2012): 202 -207.

[4] Piotr Bilski, Wieslaw Winiecki. Virtual Spectrum Analyzer Based on Data Acquisition Card, J. IEEE Transactions on Instrumentation and Measurement, 2002, (51):82-87. 